\title{
ANTI-MUSK ANTIBODY POSITIVE MYASTHENIA GRAVIS WTH CHILDHOOD ONSET
}

\author{
Diana E pure', Smaranda Nita1, Raluca I oana Teleanu' ${ }^{1,2}$ \\ 1"Dr. Victor Gomoiu” Children's Hospital, Bucharest, Romania \\ 2"Carol Davila" University of Medicineand Pharmacy, Bucharest, Romania
}

\begin{abstract}
Myasthenic syndromes are acquired diseases which unite the impairment of neuromuscular transmission with associated muscle weakness, occasional positive anti-acetylcholine or MuSK antibodies and characteristic electrodiagnostic features.

We present the case of a ten year old girl with ocular muscle weakness at onset and positive anti-MuSK antibodies, with a favorable evolution under pyridostigmine bromide.
\end{abstract}

Keywords: myasthenia, MuSK, acetylcholine, pyridostigmine, child

\section{INTRODUCTION}

Myasthenic syndromes represent a group of inherited or acquired diseases which have as a common denominator the impairment of neuromuscular transmission, resulting in various clinical patterns of muscular weakness associated with characteristic electrodiagnostic features.

Etiologically, they can be autoimmune, congenital or genetic and toxic. All of the etiopathogenic mechanisms, through different means, finally lead to neuromuscular blockade.

Autoimmune mediated Myasthenia Gravis in children can be divided, depending on the age of onset, into a neonatal transitory type and a juvenile form. The neonatal form affects children born from mothers who have anti-acetylcholine receptor (anti-AchR) antibodies but it has also been reported in mothers who have anti-MuSK antibodies. The juvenile form is classically described as manifesting after the age of ten, with a higher preponderance towards affecting girls after puberty. Ocular involvement reportedly has a higher incidence before puberty, while generalized myasthenia gravis tends to be more common after puberty (1).

The diagnosis of myasthenia relies on the suspicion raised by the characteristic history and clinical pattern and on the confirmation provided by the paraclinical tests.

Clinically, myasthenia gravis is divided, according to the Myasthenia Gravis Foundation of America Clinical Classification, into five main classes and several subclasses, the first class designating the pure ocular forms, with exclusive involvement of the extraocular muscles, while the others describing different patterns of systemic involvement, also affecting other different muscle groups: facial, bulbar, respiratory, limbs. Muscle weakness with diurnal variability is the clinical hallmark of myasthenia, with progressive fatigability with muscle use during activity. The most frequent clinical findings are bilateral asymmetric blepharoptosis and ophtaloparesis. Facial and oropharyngeal weakness result in dysarthria and dysphagia, weak cough. The symptoms can progress to respiratory failure from bulbar dysfunction or involvement of diaphragm or intercostal muscles. Limb weakness is usually more prominent proximally.

Serological assessment consists of anti-AchR antibodies, which are specific for the diagnosis of autoimmune myasthenia gravis but they are found predominantly in adult patients with generalized disease, with lower incidence of positivity in ocular 
forms. Seropositivity is more common in girls, with onset of disease after puberty (2).

Anti-AchR negative patients can be tested for Anti-MuSK antibodies. Studies show a low prevalence in children, especially in ocular forms $(3,4)$ and in children younger than 11 years. Also, thymic pathology seems to be less associated with antiMuSK antibodies positive cases (5).

Other biological studies include striated muscle antibodies, anti-lipoprotein-related protein 4 (LRP4) antibodies and thyroid assessment tests.

Electrophysiological testing can demonstrate a neuromuscular junction transmission deffect by showing a decremental response greater than 10 percent of the compound muscle action potential (CMAP) to repetitive stimulation of a motor nerve. Single-fiber electromyography is a useful method of investigation but has a lower chance of being successfully carried out in children, being a lenghty procedure that requires patient cooperation.

Treatment options in myasthenia gravis consist in: acetylcholine esterase inhibitors, thymectomy, corticosteroids and other immunosuppresive agents although the latter have not been extensively studied in children. Intravenous immunoglobulin and plasmapheresis are reserved for refractory cases or for myasthenic crisis.

\section{CASE REPORT}

We present the case of a ten year and one month old female patient, known with no significant family of personal history, who was admitted to our hospital for recurrent episodes of diplopia and strabismus. The history reveals an onset two months prior to admission with paroxysmal intermittent diplopia, observed after watching television, which persisted for about twenty-four hours. There was no history of recent viral illness or immunization. A second episode took place in a one month interval and associated diplopia and bilateral esotropia, symptoms which were preceded by an episode of vomiting. The ophthalmologic evaluation raised the suspicion of sixth nerve palsy. An infectious etiology was first suspected but was ruled out in an infectious disease center. Cerebral and contrastenhanced orbit MRI were performed, which were normal, excluding an intracranial mass.

In the following month, the child had three more episodes of paroxysmal diplopia with alternative left/right esotropia or exotropia, with duration between one hour and five days.

The neurological examination showed asymmetric limited abduction of the eyes, bilateral asymmetric mild blepharoptosis, with no involvement of other muscles (Fig. 1, 2, 3).

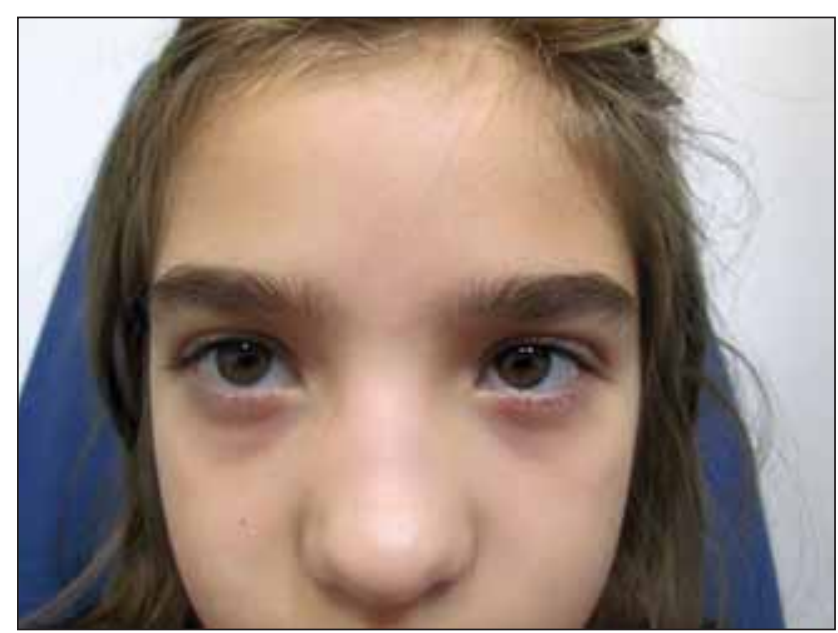

FIGURE 1. E.B, 10 years old

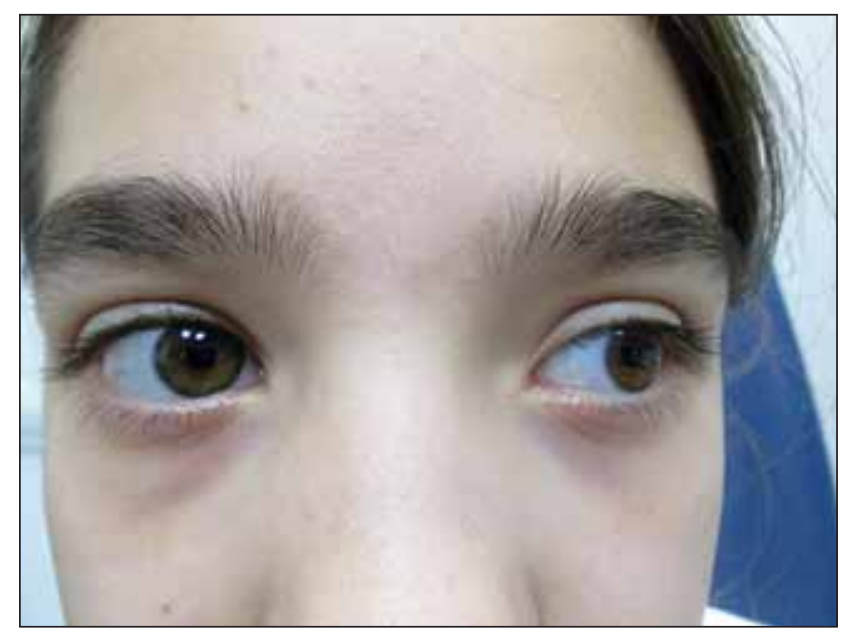

FIGURE 2. E.B, 10 years old

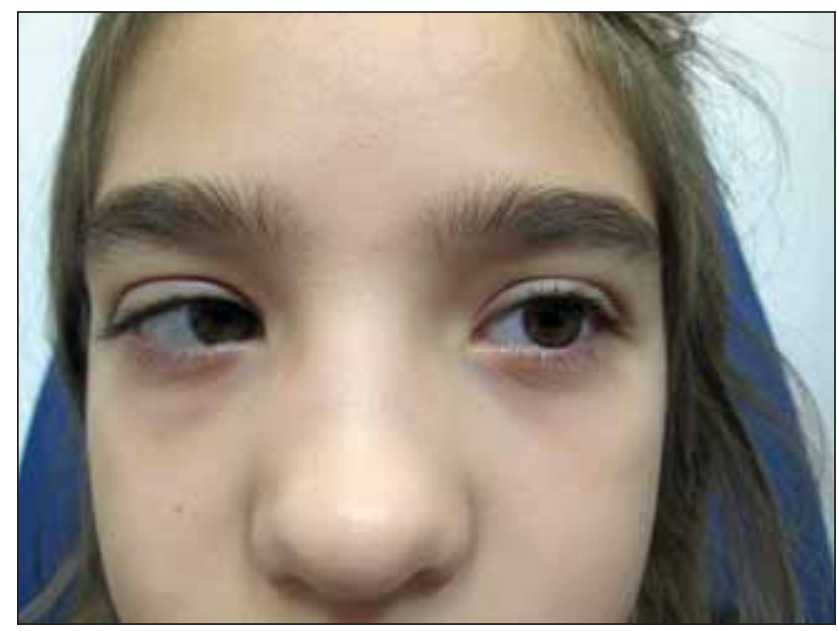

FIGURE 3. E.B, 10 years old

We performed an ice-pack test, which was positive (Fig. 4, 5). 


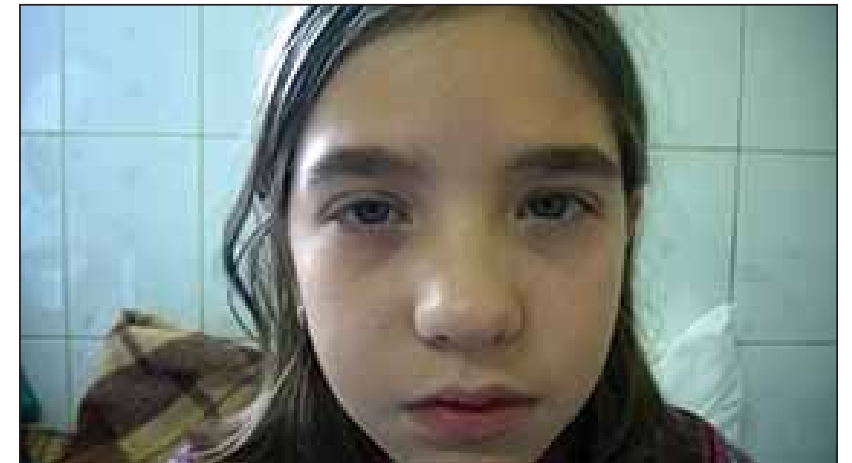

FIGURE 4. Ice-pack test- before

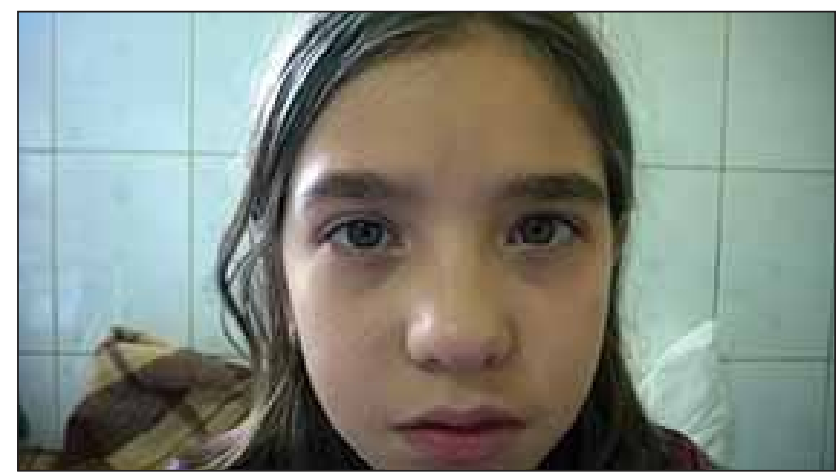

FIGURE 5. Ice-pack test: after

Anti-AchR antibodies were negative. However, anti-MuSK antibodies were positive $(2.06 \mathrm{nmol} / \mathrm{l}$, normal under $0,05 \mathrm{nmol} / \mathrm{l})$.

\section{REFERENCES}

1. Sommer N., Melms A., Weller M., Dichgans J. Ocular myasthenia gravis. A critical review of clinical and pathophysiological aspects, Doc Ophthalmol, 1993; 84(4):309-33

2. Oger J., Frykman H. An update on laboratory diagnosis in myasthenia gravis, Clinica Chimica Acta 2015, 444: 126-131

3. Caress J.B., Hunt C.H., Batish S.D. Anti-MuSK myasthenia gravis presenting with purely ocular findings, Arch Neurol 2005; 62:1002-1003
The mediastinum CT showed homogenous persistent, moderately iodophilic thymus, with tissue densities, extending over the brachiocephalic trunk.

After a positive therapeutic trial, the patient was started on pyridostigmine bromide, $150 \mathrm{mg} /$ day divided in four doses, with a favorable evolution, with rare episodes of exotropia or esotropia.

After six months of treatment, the neurological examination revealed no signs of generalized muscle weakness.

\section{DISCUSSION AND CONCLUSIONS}

The case presented illustrates the particular appearance of autoimmune myasthenia with positive anti-MuSK antibodies in a girl of prepubertal age, with pure ocular form at the moment of diagnosis, which showed favorable evolution under pyridostigmine.

The child is periodically monitored for signs of progression towards involvement of bulbar muscles or generalized weakness. Corticotherapy and immunosuppressive drugs are future possible therapeutic alternatives, in case of disease progression.

4. Gungor-Tuncer O., Orhan E.K., Yilmaz V., Parman Y., Oflazer P., Saruhan-Direskeneli G., Deymeer F. Prepubertal anti-Musk positive myasthenia gravis with long remission, Neuromuscular Disorders 2014, 24: 36-39

5. Lauriola L., Ranelletti F., Maggiano N., Guerriero M., Punzi C., Marsili F., Bartoccioni E., Thymus A. Changes in anti-MuSKpositive and -negative myasthenia gravis, Evoli, Neurology 2005; 64(3):536. 